\title{
Wall- and Hybridisation-Selective Synthesis of Nitrogen-Doped Double-Walled Carbon Nanotubes
}

Marco Carini, ${ }^{[a]}$ Lei Shi, ${ }^{[b, c]}$ Thomas W. Chamberlain, ${ }^{[\mathrm{d}, e]}$ Meng Liu, ${ }^{[f]}$ Giovanni Valenti, ${ }^{[f]}$ Manuel Melle-Franco, ${ }^{[g]}$ Francesco Paolucci, ${ }^{[f]}$ Andrei N. Khlobystov, ${ }^{*[d]}$ Thomas Pichler, ${ }^{*[b]}$ and Aurelio Mateo-Alonso*[a,g]

[a] POLYMAT, University of the Basque Country UPV/EHU. Avenida de Tolosa 72, 20018 Donostia-San Sebastian, Spain. E-mail: amateo@polymat.eu

[b] University of Vienna, Faculty of Physics. Strudlfogasse 4, 1090 Vienna, Austria. E-mail: thomas.pichler@univie.ac.at

[c] School of Materials Science \& Engineering, Sun Yat-sen University. Guangzhou 510275, Guangdong, P. R. China

[d] School of Chemistry, University of NottinghamUniversity Park, Nottingham, UK. E-mail: Andrei.Khlobystov@nottingham.ac.uk

[e] School of Chemistry, University of Leeds. Leeds, UK

[f] Università di Bologna. Dipartimento di Chimica "Giacomo Ciamician". Via Selmi 2, 40126 Bologna, Italy.

[g] CICECO - Aveiro Institute of Materials, Department of Chemistry, University of Aveiro. 3810-193 Aveiro, Portugal

[f] Ikerbasque, Basque Foundation for Science. Bilbao, Spain 
Abstract: Controlled nitrogen-doping is a powerful methodology to modify the properties of carbon nanostructures and produce functional materials for electrocatalysis, energy conversion and storage, and sensing, among others. Herein, we report a wall- and hybridisation-selective synthetic methodology to produce double-walled carbon nanotubes with an inner tube doped exclusively with graphitic $\mathrm{sp}^{2}$-nitrogen atoms. Our measurements shed light on the fundamental properties of nitrogen-doped nanocarbons opening the door for developing their potential applications. 
Controlled substitutional nitrogen-doping is a powerful methodology to modify the properties of carbon nanostructures. ${ }^{[1-14]}$ The synthesis and characterisation of nitrogen-doped fullerenes, carbon nanotubes, graphene and graphene nanoribbons have revealed that nitrogen-doped carbon nanostructures are promising materials for electrocatalysis, ${ }^{[2-9,11,13-14]}$ energy conversion and storage, ${ }^{[2,4-7,10,13-14]}$ and sensing, ${ }^{[13-14]}$ among others. The synthesis of nitrogendoped nanocarbons still presents challenges for controlling: (i) the inclusion percent and the distribution of nitrogen within the graphitic lattice; (ii) the bonding of the nitrogen atoms, as principally three types of nitrogen (pyridinic, graphitic, and pyrrolic) can exist; (iii) wall-selectivity, in the case of layered nanocarbons (e.g. double-walled and multi-walled carbon nanotubes, or stacked layers of graphene). Among the above-mentioned challenges, wall-selectivity has received little attention ${ }^{[15-17]}$ and provides numerous opportunities for establishing and understanding the fundamental properties of nitrogen-doped nanocarbons and for developing their potential applications. ${ }^{[3]}$ 


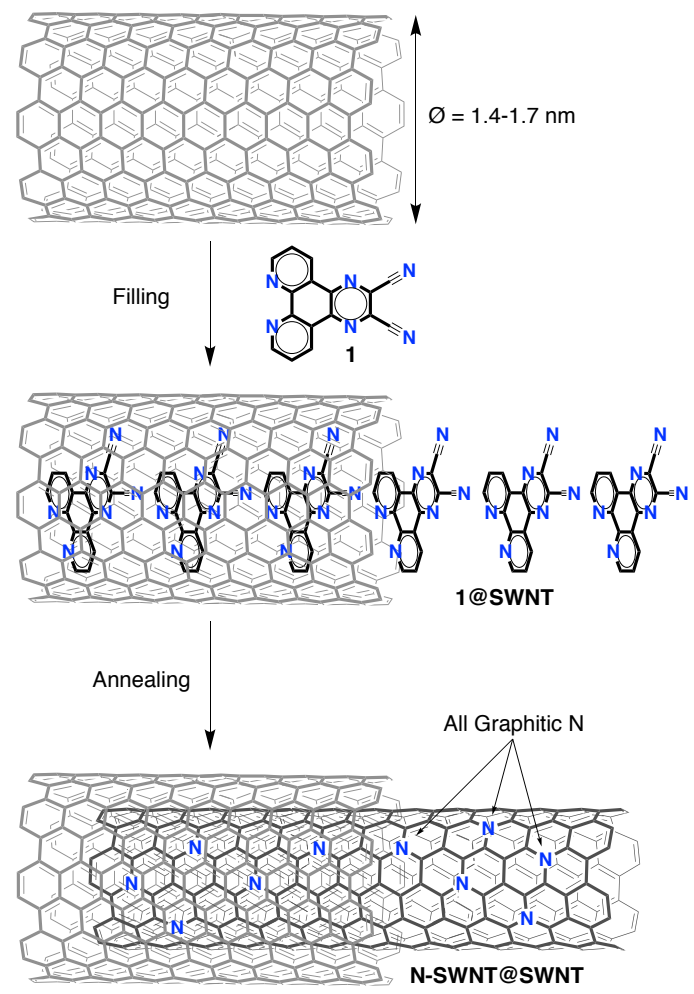

Scheme 1. Synthetic approach for the preparation of coaxial N-SWNT@SWNT. 
Herein, we report a wall- and hybridisation-selective synthetic methodology to produce double-walled carbon nanotubes (DWNT) with an inner tube doped exclusively with graphitic nitrogen atoms (Scheme 1). Such coaxial carbon nanotubes have been prepared in two steps by using a nitrogen-rich polycyclic aromatic hydrocarbon, namely dicyanopyrazophenanthroline 1 , as feedstock and empty single-walled carbon nanotubes (SWNT) as reaction vessels. Firstly, dicyanopyrazophenanthroline $\mathbf{1}$ is sublimed into the internal cavity of SWNT. Then, the filled SWNTs (1@SWNT) are exposed to an electron beam or thermally treated so the encapsulated dicyanopyrazophenanthroline 1 converts into a nanotube to produce DWNT with an inner nanotube doped with nitrogen $(\mathbf{N}$ SWNT@SWNT). The graphitic nitrogen content in the inner tube can be controlled with the annealing temperature to reach values up to $100 \%$, as demonstrated by a combination of Raman spectroscopy, high-resolution transmission electron microscopy (HR-TEM), energy-dispersive X-ray spectroscopy (EDX), X-ray photoemission spectroscopy (XPS) and cyclic voltammetry.

Dicyanopyrazophenanthroline 1 was selected as feedstock because of the high $\mathrm{C} / \mathrm{N}$ ratio $(16 / 6)$, the fact that all the nitrogen atoms are already incorporated in the $\pi$ framework as $s p^{2}$ or $s p$ nitrogen; and its ability to sublime. Dicyanopyrazophenanthroline 1 was synthesised following a reported procedure ${ }^{[18]}$ and then to ensure a high level of purity it was further purified by chromatography on alumina and reprecipitation (see experimental procedures in supporting information). 
Opened SWNT were filled with 1 by heating a mixture of the compound with SWNT in $10^{-6}$ mbar vacuum at $120^{\circ} \mathrm{C}$. HR-TEM imaging confirmed that the molecules become encapsulated inside the SWNT, yielding 1@SWNT (Figure 1). The molecules of 1 are disordered within the cavity of the SWNT, although in some areas, the molecules appear to stack within the nanotube cavity (Figure 1 top zoomed image). Extended exposure to the electron beam resulted in the transformation of the molecules within the nanotube into a new nanotube, thus forming a DWNT (N-SWNT@SWNT). The formation of N-SWNT@SWNT is evidenced by the presence of long continuous structures coaxial to the external tube, terminated with internal caps (Figure 1 bottom zoomed image).

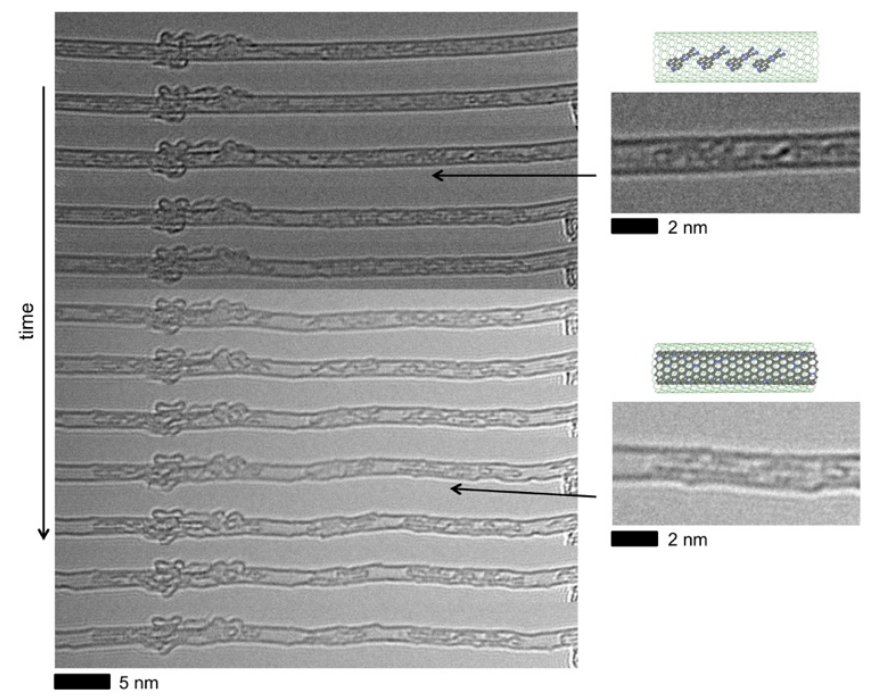

Figure 1. HR-TEM of 1@SWNT and formation of N-SWNT@SWNT over time upon exposure to the $100 \mathrm{keV}$ electron beam. The zoomed images show the disordered molecules inside the cavity of the SWNT (top) and the newly formed internal nanotube (bottom). 
HR-TEM imaging clearly showed the propensity of 1 to convert into a nanotube. To investigate in more detail the structural features of N-SWNT@SWNT, bulk filling and annealing experiments were carried out with SWNT with diameters 1.4$1.7 \mathrm{~nm}$ in order to obtain sufficient material for structural characterisation. The filling of SWNT with 1 and the transformation process of $1 @$ SWNT into NSWNT@SWNT by annealing at different temperatures was monitored by resonance Raman spectroscopy and the samples were further analysed by HRTEM, energy dispersive X-ray spectroscopy (EDX) and by X-ray photoemission spectroscopy (XPS).

Figure 1 shows the Raman spectra of the pristine SWNT, 1@SWNT and NSWNT@SWNT formed by annealing at different temperatures. In all the spectra, the G-band, D-band, 2D-band and radial breathing mode (RBM) bands typical of SWNT can be clearly observed. In the spectrum of 1@SWNT, additional Raman bands corresponding to those of the spectra of 1 were detected (Figure S1). The Raman spectra show the appearance of new bands in the RBM region, of a new D-band and of a new contribution (shoulder) in the 2D-band with the increasing annealing temperatures. In the case of the samples obtained at 1300 and 1400 ${ }^{\circ} \mathrm{C}$, the new bands in the RBM region are consistent with the formation of an internal tube within the SWNT cavity (N-SWNT@SWNT). Furthermore, the Raman spectrum of $1 @$ SWNT exhibits a nitrile band consistent with the structure of precursor 1 that disappears upon annealing (Figure S1). 


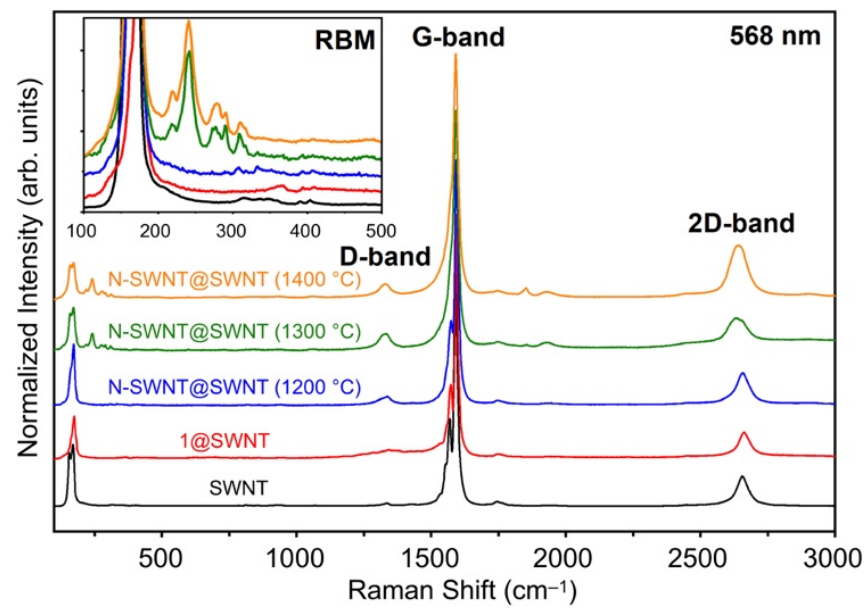

Figure 2. Resonant Raman spectra of 1@SWNT annealed at different temperatures.

To confirm that the precursor molecules are inside SWNT and that the new inner nanotube grows coaxially to the host nanotube, 1@SWNT and N-SWNT@SWNT were investigated by HR-TEM. The images of 1@SWNT evidenced excellent filling rates of molecules inside the SWNT cavities (Figures 3a and S2). Also, the images of N-SWNT@SWNT samples obtained at $1300^{\circ} \mathrm{C}$ and $1400^{\circ} \mathrm{C}$ showed long continuous structures within the SWNT consistent with the formation of wellstructure inner nanotubes (Figures $3 b$ and S3). In addition, caps on internal nanotubes can be clearly observed similar to those formed under the electron beam. EDX provide the first evidence of incorporation of nitrogen in the nanotube with a new emerging peak of nitrogen that is not observed in the empty SWNT sample (Figure 3c).

The nitrogen inclusion percentage and bonding on 1@SWNT and NSWNT@SWNT was investigated by XPS (Figures 3d and S4; Table 1). The N1s region of the XPS spectrum of $1 @$ SWNT indicates a $10.6 \%$ of nitrogen present, distributed between pyridinic, nitrilic and graphitic nitrogen. The percentage of pyridinic and nitrilic nitrogen is higher than that of graphitic nitrogen, which agrees 
with the structure of molecule 1. The presence of graphitic nitrogen on 1@SWNT was ascribed to the partial decomposition of 1 within the SWNT because of the high temperature used $\left(300^{\circ} \mathrm{C}\right)$ during the filling and cleaning processes. Importantly, the XPS spectrum of N-SWNT@SWNT obtained after annealing at $1300{ }^{\circ} \mathrm{C}$ confirms the incorporation of $0.9 \%$ of nitrogen and shows the disappearance of nitrilic nitrogen - in agreement with Raman - and a higher proportion of graphitic nitrogen versus pyridinic nitrogen. The measured nitrogen incorporation indicates $2.4 \%$ of nitrogen in the inner tube (Table 1 and Table S1), which equates to two or more nitrogen atoms per nm in length. Remarkably, the N-SWNT@SWNT obtained at $1400{ }^{\circ} \mathrm{C}$ shows only a contribution of graphitic nitrogen, while the total nitrogen inclusion percentage is virtually unchanged, which imply the formation of an internal nanotube doped exclusively with graphitic nitrogen.
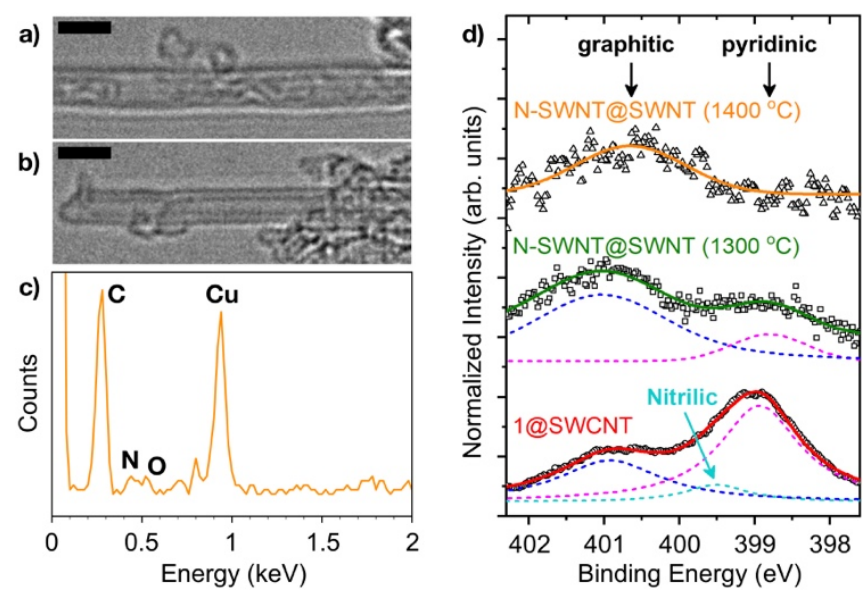

Figure 3. HR-TEM of a) 1@SWNT and b) N-SWNT@SWNT. Scale bar=2 nm b) EDX of N-SWNT@SWNT indicating the presence of N. c) XPS of 1@SWNT and N-SWNT@SWNT obtained at different temperatures showing the presence and hydbridisation of $\mathrm{N}$ atoms. 
Table 1. Content and hybridisation of nitrogen in the different nanotubes $(\% \mathrm{~N})$.

\begin{tabular}{|c|c|c|c|c|c|}
\hline Sample & Total & $\begin{array}{l}\text { Inner } \\
\text { Tube }\end{array}$ & Nitrilic ${ }^{[b]}$ & Pyridinic $^{[\mathrm{b}]}$ & Graphitic $^{[b]}$ \\
\hline 1@SWNT & $10.6 \%$ & - & $8.8 \%$ & $60.6 \%$ & $30.6 \%$ \\
\hline $\begin{array}{l}\text { N-SWNT@SWNT } \\
\left(1300^{\circ} \mathrm{C}\right)\end{array}$ & $0.9 \%$ & $2.4 \%$ & $0 \%$ & $16.2 \%$ & $83.8 \%$ \\
\hline $\begin{array}{l}\text { N-SWNT@SWNT } \\
\left(1400^{\circ} \mathrm{C}\right)\end{array}$ & $0.8 \%$ & $2.4 \%$ & $0 \%$ & $0 \%$ & $100.0 \%$ \\
\hline
\end{tabular}

[a] Estimated assuming an external tube diameter of $1.4 \mathrm{~nm}$, a $100 \%$ filling (Table S1). [b] Measured by XPS.

The electrochemical properties of N-SWNT@SWNT were investigated by cyclic voltammetry in a rotating disk electrode (Figures $4 a$ and S5; Table S2). The voltammetric curves in an argon saturated aqueous $0.1 \mathrm{M} \mathrm{KOH}$ solution show no redox processes, but in an oxygen saturated solution, the voltammograms reveal a reduction wave characteristic of the oxygen reduction reaction, $\left.{ }^{2-4,6-7,} 9,11,13-14\right]$ in which the presence and bonding of nitrogen atoms are directly linked to the electrode potentials. ${ }^{[19-25]}$ The glassy carbon (GC) electrodes containing $\mathbf{N}$ SWNT@SWNT show more anodic potentials at $1 \mathrm{mAcm}^{-2}$ than those containing pristine SWNT (0.564 V vs NHE). Furthermore, the potential at $1 \mathrm{mAcm}^{-2}$ of the N-SWNT@SWNT $\left(1300^{\circ} \mathrm{C}\right)$ with $83.8 \%$ of graphitic nitrogen $(0.580$ V vs NHE) is more anodic than that of $\mathbf{N}$-SWNT@SWNT $\left(1400^{\circ} \mathrm{C}\right)$ with $100.0 \%$ of graphitic nitrogen $(0.685 \mathrm{~V}$ vs NHE). The voltammograms confirm that the presence of graphitic nitrogen shifts the potential to more anodic values and also show that the nitrogen atoms of the internal nanotube are able to modulate the electrochemical properties even if they are encapsulated by the external carbon nanotube. 
a)

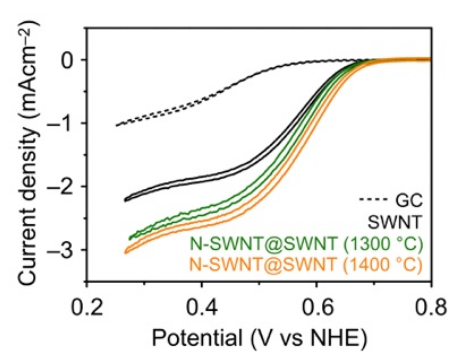

b)

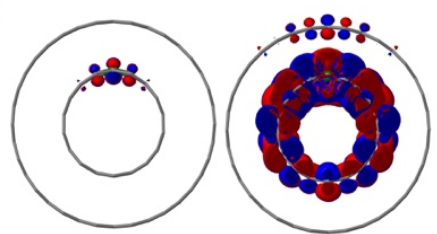

Figure 4. a) Effect of nitrogen bonding on the electrochemical properties of $\mathbf{N}$ SWNT@SWNT. CVs of a bare GC electrode, SWNT, N-SWNT@SWNT (1300 $\left.{ }^{\circ} \mathrm{C}\right), \mathbf{N}$-SWNT@SWNT $\left(1400{ }^{\circ} \mathrm{C}\right) \mathrm{O}_{2}$-saturated $0.1 \mathrm{M} \mathrm{KOH}$ aqueous solution (scan rate: $10 \mathrm{mV} \mathrm{s}$; rotating rate: $1600 \mathrm{rpm}$ ). b) HOMO orbitals of representative N-SWNT@SWNT $((9,0) @(18,0)$ with $2.3 \% \mathrm{~N})$ with $0.02 \mathrm{e}^{-} / \mathrm{A}^{3}$ (left) and $0.001 \mathrm{e}^{-} / \mathrm{A}^{3}$ (right) isosurfaces.

To investigate if the internal nitrogen-doped nanotube is able transduce some of its properties across the external one, computational modelling was performed on semiconducting and metallic DWNT models with different doping degrees in the internal tube (Table S3). First, the extra electron of nitrogen at the doping levels measured yields metallic DWNTs in all cases through a direct electron transfer mechanism (Table S4). In addition, an analysis of the electron distribution indicates that there is a direct charge transfer from the doped internal tube to the external tube and that this transfer increases with $\mathrm{N}$ content (Table S4). Secondly, the density of states (DOS) shows very little changes in the states of the external undoped SWNT, in contrast to the electronic structure of the internal nanotubes, which is extensively altered due to the presence of nitrogen (Figures S6 and S7). The analysis of the frontier orbitals shows that there are levels from the internal and external tubes in a similar range of energies and that 
the nitrogen atoms area and the external tubes are coupled (Figure S8). This can be seen in the HOMO molecular orbital, showing an enhanced electronic density near the nitrogen atoms in the external tube (Figure 4b). The electronic doping of the external SWNT induced by the internal tube creates potential differences (Table S4), which are consistent with the ones observed by the cyclic voltammetry.

To conclude, we have reported a wall- and hybridisation-selective synthetic methodology to prepare nitrogen-doped double-walled carbon nanotubes (NSWNT@SWNT). A nitrogen-rich polycyclic aromatic hydrocarbon (1) has been inserted into the cavity of SWNT and then by exposing the samples to an electron beam or to annealing temperatures over $1300^{\circ} \mathrm{C}$, the encapsulated molecules are transformed into a nitrogen-doped carbon nanotube within the SWNT cavity. The transformation can be monitored by Raman spectroscopy and HR-TEM. XPS confirms the inclusion of $\sim 1 \%$ of nitrogen in the newly formed carbon nanotube and also illustrate that the hybridisation of the nitrogen atoms can be controlled with the annealing temperature. For instance, thermal annealing at $1400{ }^{\circ} \mathrm{C}$ produce double-walled carbon nanotubes with an inner tube doped exclusively with graphitic nitrogen, while annealing at $1300^{\circ} \mathrm{C}$ produce double-walled carbon nanotubes with an inner tube doped with both pyridinic and graphitic nitrogen. Electrochemical studies and theoretical calculations confirm that the internal nitrogen-doped nanotube is able transduce some of its properties across the external nanotube. Overall this method enables the synthesis of nitrogen-doped nanocarbons with an unprecedented level of control that allows investigating and harnessing their functional properties. 


\section{Acknowledgements}

M.C. and A.M.A acknowledge support of the Basque Science Foundation for Science (Ikerbasque), POLYMAT, the University of the Basque Country (Grupo de Investigación GIU17/054 and SGIker), Gobierno de España (Ministerio de Economía y Competitividad CTQ2016-77970-R) and Gobierno Vasco (BERC program). This project has received funding from the European Research Council (ERC) under the European Union's Horizon 2020 research and innovation programme (grant agreement $n^{\circ}$ 722951). M.M.F. acknowledge support of the Portuguese Foundation for Science and Technology (FCT), under the projects PTDC/FIS-NAN/4662/2014, IF/00894/2015, and FCT Ref. UID/CTM/50011/2019 for CICECO - Aveiro Institute of Materials. T.W.C. and A.N.K. acknowledge support of the Nanoscale and Microscale Research Centre (nmRC), University of Nottingham, for access to microscopy equipment. 


\section{References}

[1] T. Susi, P. Ayala, in Carbon Nanomaterials for Advanced Energy Systems (Eds.: W. Lu, J.-B. Baek, L. Dai), John Wiley \& Sons, Inc., 2015, pp. 133-161.

[2] S. Zhao, D.-W. Wang, R. Amal, L. Dai, Adv. Mater. 2019, 31, 1801526.

[3] C. Hu, L. Dai, Adv. Mater. 2019, 31, 1804672.

[4] M. Antonietti, M. Oschatz, Adv. Mater. 2018, 30, 1706836.

[5] J. Zhang, G. Chen, K. Müllen, X. Feng, Adv. Mater. 2018, 30, 1800528.

[6] J. Mahmood, M. A. R. Anjum, J.-B. Baek, Adv. Mater. 2018, 0, 1805062.

[7] D. Liu, K. Ni, J. Ye, J. Xie, Y. Zhu, L. Song, Adv. Mater. 2018, 30, 1802104.

[8] H. Jin, C. Guo, X. Liu, J. Liu, A. Vasileff, Y. Jiao, Y. Zheng, S.-Z. Qiao, Chem. Rev. 2018, 118, 6337-6408.

[9] X. Liu, L. Dai, Nat. Rev. Mater. 2016, 1, 16064.

[10] N. A. Kumar, J.-B. Baek, Nanotechnology 2015, 26, 492001.

[11] L. Dai, Y. Xue, L. Qu, H.-J. Choi, J.-B. Baek, Chem. Rev. 2015, 115, 48234892.

[12] A. Mateo-Alonso, Chem. Soc. Rev. 2014, 43, 6311-6324.

[13] Y. Zhang, J. Zhang, D. S. Su, ChemSusChem 2014, 7, 1240-1250.

[14] P. Ayala, R. Arenal, M. Rümmeli, A. Rubio, T. Pichler, Carbon 2010, 48, $575-586$.

[15] Yang, Xu, A. Tomita, T. Kyotani, J. Am. Chem. Soc. 2005, 127, 8956-8957.

[16] A. Lopez-Bezanilla, J. Phys. Chem. C 2014, 118, 1472-1477.

[17] G.-L. Tian, Q. Zhang, B. Zhang, Y.-G. Jin, J.-Q. Huang, D. S. Su, F. Wei, Adv. Funct. Mater. 2014, 24, 5956-5961.

[18] A. Ambroise, B. G. Maiya, Inorg. Chem. 2000, 39, 4264-4272. 
[19] N. Wang, B. Lu, L. Li, W. Niu, Z. Tang, X. Kang, S. Chen, ACS Catalysis 2018, 8, 6827-6836.

[20] C. V. Pham, B. Britton, T. Böhm, S. Holdcroft, S. Thiele, Adv. Mater. Interfaces 2018, 5, 1800184.

[21] D. Guo, R. Shibuya, C. Akiba, S. Saji, T. Kondo, J. Nakamura, Science 2016, 351, 361-365.

[22] H. B. Yang, J. Miao, S.-F. Hung, J. Chen, H. B. Tao, X. Wang, L. Zhang, R. Chen, J. Gao, H. M. Chen, L. Dai, B. Liu, Science Adv. 2016, 2, e1501122.

[23] L. Lai, J. R. Potts, D. Zhan, L. Wang, C. K. Poh, C. Tang, H. Gong, Z. Shen, J. Lin, R. S. Ruoff, Energy Environ. Sci. 2012, 5, 7936-7942.

[24] C. V. Rao, C. R. Cabrera, Y. Ishikawa, J. Phys. Chem. Lett. 2010, 1, 26222627.

[25] K. Gong, F. Du, Z. Xia, M. Durstock, L. Dai, Science 2009, 323, 760-764. 